\title{
Programmed cell death 4 and BCR-ABL fusion gene expression are negatively correlated in chronic myeloid leukemia
}

\author{
XIA ZHANG ${ }^{*}$, RIMING LIU* ${ }^{*}$ BAOHUA HUANG, XIAOLU ZHANG, \\ WEIJUAN YU, CUIXIA BAO, JIE LI and CHENGMING SUN
}

Department of Laboratory Medicine, Yuhuangding Hospital of Qingdao University, Yantai, Shandong 264000, P.R. China

Received April 14, 2015; Accepted July 20, 2016

DOI: $10.3892 / \mathrm{ol} .2016 .4942$

\begin{abstract}
Programmed cell death 4 (PDCD4) is a tumor suppressor that inhibits carcinogenesis, tumor progression and invasion by preventing gene transcription and translation. Downregulation of PDCD4 expression has been identified in multiple types of human cancer, however, to date, the function of PDCD4 in leukemia has not been investigated. In the present study, PDCD4 mRNA and protein expression was investigated in 50 patients exhibiting various phases of chronic myeloid leukemia (CML) and 20 healthy individuals by reverse transcription-quantitative polymerase chain reaction and western blot analysis. PDCD4 expression and cell proliferation was also investigated following treatment with the tyrosine kinase inhibitor, imatinib, in K562 cells. The results demonstrated that PDCD4 mRNA and protein expression was decreased in all CML samples when compared with healthy controls, who expressed high levels of PDCD4 mRNA and protein. No significant differences in PDCD4 expression were identified between chronic phase, accelerated phase and blast phase CML patients. In addition, PDCD4 expression was negatively correlated with BCR-ABL gene expression $(r=-0.6716$; $\mathrm{P}<0.001)$. Furthermore, K562 cells treated with imatinib exhibited significantly enhanced PDCD4 expression. These results indicate that downregulation of PDCD4 expression may exhibit a critical function in the progression and malignant proliferation of human CML.
\end{abstract}

\section{Introduction}

Chronic myeloid leukemia (CML) is a myeloproliferative disease characterized by the presence of the Philadelphia

Correspondence to: Mr. Chengming Sun or Mrs. Jie Li, Department of Laboratory Medicine, Yuhuangding Hospital of Qingdao University, 20 Yuhuangding East Road, Yantai, Shandong 264000, P.R. China

E-mail: sunchengming1972@163.com

E-mail: 1ijiehao77@sina.com

*Contributed equally

Key words: programmed cell death 4, tumor suppressor, expression, chronic myeloid leukemia, BCR-ABL
$(\mathrm{Ph})$ chromosome, which is formed by a $\mathrm{t}(9 ; 22)(\mathrm{q} 34 ; \mathrm{q} 11)$ balanced reciprocal translocation (1). The $\mathrm{Ph}$ chromosome translocation generates the BCR-ABL oncogene that encodes for the BCR-ABL oncoprotein, which exhibits constitutively active tyrosine kinase activity that promotes the growth of leukemic cells (2). CML patients in the chronic phase (CP) that are treated with tyrosine kinase inhibitors (TKIs) achieve a significant effect (1). However, a significant percentage of patients develop TKI resistance and disease recurrence (3), which involves a variety of cellular mechanisms. Therefore, identifying molecules that are involved in the development and progression of CML may provide novel therapeutic targets for CML treatment.

Programmed cell death 4 (PDCD4) is a novel tumor suppressor that inhibits tumor growth via suppression of protein translation by binding to eukaryotic initiation factor (EIF) 4A via two MA-3 domains, which are highly homologous to eIF4G (4). PDCD4 also suppresses translation elongation by combining directly with target gene coding regions, such as c-myb (5) and A-myb (6). PDCD4-deficient mice exhibit a significantly reduced life span and develop spontaneous lymphomas with frequent metastasis to the liver and kidneys (7). PDCD4 transgenic mice exhibit resistance to tumor promotion and progression in response to a multistage carcinogenesis regimen (8). Additionally, decreased or absent PDCD4 expression has been identified in several types of human cancer, including lung (9), hepatocellular (10), colon (11), glioma (12) and ovarian cancers (13). Overexpression of PDCD4 suppresses tumor phenotypes by inhibiting activator protein-1 (AP-1) transactivation in JB6 cells (14). PDCD4 overexpression and has also been demonstrated to inhibit invasive capacity in colon RKO cells (15), inhibit tumor cell intravasation (16) and suppress malignant phenotypes of human ovarian cancer (13). Furthermore, PDCD4 knockdown significantly promotes invasion and activates both $\beta$-catenin/T cell factor (Tcf) and AP-1-dependent transcription (17). Additionally, PDCD4 knockdown leads to increased Snail expression and subsequent downregulation of E-cadherin resulting in the activation of catenin/Tcf-dependent transcription and the expression of c-Myc and urokinase receptor (18).

Taken together, these findings indicate that PDCD4 presents a potential target in the diagnosis and treatment of neoplasms. However, whether PDCD4 is involved in human hematologic neoplasms remains unclear. In the present study, 
PDCD4 expression levels in CML patients were evaluated using reverse transcription-quantitative polymerase chain reaction (RT-qPCR) and western blot analysis.

\section{Materials and methods}

Patients and samples. A total of 50 bone marrow aspirate samples were obtained from patients diagnosed with CML, according to the World Health Organization guidelines (19), at Yuhuangding Hospital of Qingdao University (Yantai, China) between June 2012 and September 2014. The CML patient sample included 23 females and 27 males with a mean age ( \pm standard deviation) of 41.94 years $( \pm 14.13$ years $)$. Patients were divided into the following three groups depending on their clinical and laboratory data: into $30 \mathrm{CP}(\mathrm{n}=30)$, accelerated phase (AP; $n=10)$ and blast phase $(B P ; n=10)$. The criteria for diagnosis of CML-CP was the presence of $\mathrm{t}(9 ; 22)$ or the BCR-ABL fusion gene, $<10 \%$ bone marrow blasts, and does not satisfy the diagnostic criteria of CML-AP or CML-BP. Peripheral blood samples were also obtained from 20 healthy individuals, which served as the control group. Patient characteristics are listed in Table I. The final protocol for the use of patient samples in the present study was approved by the local Institutional Review Board of Qingdao University and informed consent was obtained from all patients and controls.

Cell lines and treatment. K562 cells were purchased from the Shanghai Cell Bank of Chinese Academy of Sciences (Shanghai, China) and cultured in RPMI-1640 medium (Gibco; Thermo Fisher Scientific, Waltham, MA, USA) supplemented with $10 \%$ heat-inactivated fetal bovine serum (Gibco; Thermo Fisher Scientific,), $1 \%$ penicillin and streptomycin at $37^{\circ} \mathrm{C}$ in a humidified incubator with $5 \% \mathrm{CO}_{2}$. A total of $2 \times 10^{5}$ cells/ well were seeded in 6-well plates and treated with 0.5 and $1 \mu \mathrm{M}$ imatinib (LC Laboratories, Woburn, MA, USA) for 6, 24 and 48 h. Next, cells were harvested and washed once with phosphate-buffered saline and subjected to RT-PCR and immunoblotting analyses. Experiments were performed in triplicate.

MTT assay. A total of $8 \times 10^{3}$ cells/well were seeded into 96-well plates and treated with 0.5 and $1 \mu \mathrm{M}$ imatinib. After 6, 24 and $48 \mathrm{~h}$ of imatinib treatment, $20 \mu \mathrm{l}$ MTT reagent $(5 \mathrm{mg} / \mathrm{ml}$; Sigma-Aldrich; Merck Millipore, Darmstadt, Germany) was added to each well and incubated in the dark for $4 \mathrm{~h}$ at $37^{\circ} \mathrm{C}$. After 4 h, $100 \mu$ dimethyl sulfoxide was added to each well and the absorbance was determined using a Benchmark Plus Microplate Spectrophotometer (Bio-Rad Laboratories, Inc., Hercules, CA, USA) at $570 \mathrm{~nm}$. Cell proliferation of treated cells was calculated from the average optical density at $570 \mathrm{~nm}$ values compared to that of untreated cells. Experiments were performed in triplicate.

RNA isolation and RT-qPCR. RNA was extracted from isolated peripheral blood mononuclear cells using a modified TRIzol one-step extraction method (Invitrogen; Thermo Fisher Scientific). RNA concentrations were determined based on the absorbance at $260 \mathrm{~nm}$. Total RNA $(2 \mu \mathrm{g})$ was reversely transcribed to cDNA using the Reverse-Transcribe Kit (Promega Corp., Madison, WI, USA). Quantitative PCR was performed using SYBR ${ }^{\circledR}$ Selected Master Mix (Applied Biosystems; Thermo Fisher Scientific) and specific primer pairs (Invitrogen; Thermo Fisher Scientific Inc.). The sequences of the sense and antisense primers were as follows: Sense, 5'-TGT AAACCCTGCAGATCCTGATAA-3' and antisense, 5'-TGG AGGATGCTGAAATCCAA-3' for PDCD4; sense, 5'-GGA GCTGCAGATGCTGACCACC-3' and antisense, 5'-TCAGAC CCTGAGGCTCAAAGTC-3' for BCR-ABL; sense, 5'-AAC GGATTTGGTCGTATTGGG-3' and antisense, 5'-CCTGGA AGATGGTGATGGGAT-3' for glyceraldehyde 3-phosphate dehydrogenase (GAPDH). The samples were denatured at $95^{\circ} \mathrm{C}$ for $10 \mathrm{~min}$, followed by 39 cycles of $95^{\circ} \mathrm{C}$ for $30 \mathrm{sec}$ and $60^{\circ} \mathrm{C}$ for $1 \mathrm{~min}$ and $65^{\circ} \mathrm{C}$ for $30 \mathrm{sec}$ to stop the reaction. Each experiment was conducted in triplicate. Relative PDCD4 expression was calculated using the $\Delta \Delta \mathrm{Cq}$ model (20). All samples were normalized to GAPDH, which served as an endogenous control.

SDS-PAGE and western blot analysis. Cells were lysed in SDS sample buffer (Beyotime Institute of Biotechnology, Shanghai, China) and the protein concentration of the homogenized lysates was measured using a bicinchoninic acid protein assay kit (Beyotime Institute of Biotechnology) and equal amounts of protein were separated on 10\% SDS-PAGE (Beyotime Institute of Biotechnology) and transferred onto polyvinylidene difluoride membranes (EMD Millipore, Billerica, MA, USA). Membranes were then blocked with 5\% skimmed milk in Trisbuffered saline containing 0.1\% Tween-20 (Beyotime Institute of Biotechnology) for $1 \mathrm{~h}$ and incubated overnight at $4^{\circ} \mathrm{C}$ with rabbit monoclonal anti-PDCD4 (catalog no. 9535S; dilution, 1:1,000; Cell Signaling Technology, Inc., Beverly, MA, USA) and human polyclonal anti- $\beta$-actin (catalog no. sc-130656; dilution, 1:2,000; Santa Cruz Biotechnology, Inc., Santa Cruz, CA, USA) antibodies. The membranes were then incubated with peroxidase-conjugated goat anti-rabbit immunoglobulin $\mathrm{G}$ secondary antibody (catalog no. sc-2004; dilution, 1:5,000; Santa Cruz Biotechnology, Inc.) for $1 \mathrm{~h}$ at room temperature. After washing with Tris-buffered saline with $0.1 \%$ Tween-20, signals were visualized with SuperSignal West Pico Chemiluminescent Substrate (Pierce Biotechnology, Inc., Rockford, IL, USA). Quantification of PDCD4 protein was normalized to $\beta$-actin using Quantity One ${ }^{\circledR}$ Version 4.3.1 (Bio-Rad Laboratories, Inc.). Western blot analysis was performed at least three times for each sample.

Statistical analysis. Data were obtained from at least three independent experiments and are presented as the mean \pm standard error of the mean. All statistical analysis was performed using SPSS 10.0 statistical software (SPSS, Inc., Chicago, IL, USA). Data was analyzed using the Student's t-test or one-way analysis of variance. Pearson's coefficient was calculated to analyze the correlation between PDCD4 and BCR-ABL expression. $\mathrm{P}<0.05$ was considered to indicate a statistically significant difference.

\section{Results}

Decreased PDCD4 $m R N A$ expression is observed in CML patients. To investigate PDCD4 expression in primary CML, PDCD4 mRNA expression was analyzed in $50 \mathrm{CML}$ patients 
Table I. Clinical parameters and PDCD4 expression in 50 CML patients and 20 healthy individuals.

\begin{tabular}{lcccc}
\hline & & \multicolumn{3}{c}{ CML patients } \\
\cline { 3 - 5 } Parameter & Healthy controls & CML-CP & CML-AP & CML-BP \\
\hline Gender, $\mathrm{n}$ & & & & 4 \\
$\quad$ Male & 10 & 13 & 5 & 6 \\
Female & 10 & 17 & 49 & 51 \\
Age, years & 36 & 49 & $32-63$ & $39-76$ \\
Median & $24-59$ & $15-71$ & $0.13 \pm 0.04$ & $0.15 \pm 0.05$ \\
Range & $1.65 \pm 0.17$ & $0.150 \pm 0.03$ & & \\
PDCD4/GAPDH $( \pm$ SEM $)$ & & & & \\
\end{tabular}

PDCD4, programmed cell death 4; CML, chronic myeloid leukemia; GAPDH, glyceraldehyde 3-phosphate dehydrogenase; CP, chronic phase; AP, accelerated phase; BP, blast phase; SEM, standard error of the mean.

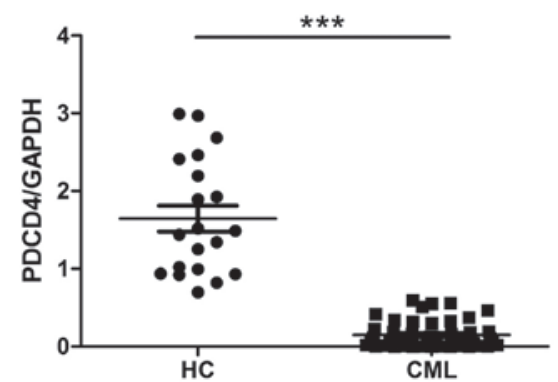

Figure 1. Expression of PDCD4 mRNA expression in 50 primary CML patients and $20 \mathrm{HCs}$ was analyzed by reverse transcription-quantitative polymerase chain reaction. PDCD4 mRNA expression was significantly higher in HCs compared with CML patients. ${ }^{* * * *} \mathrm{P}<0.001$. PDCD4, programmed cell death 4; GAPDH, glyceraldehyde 3-phosphate dehydrogenase CML, chronic myeloid leukemia; HC, healthy controls.

and 20 healthy controls by RT-qPCR. As shown in Fig. 1, high levels of PDCD4 mRNA expression were observed in the 20 healthy control samples, however, all CML patients exhibited extremely low PDCD4 expression. Furthermore, the differences between PDCD4 expression in three phases of CML were analyzed, however, no significant differences in PDCD4 mRNA expression were identified in CML-CP, CML-AP and CML-BP patients (Table I).

Decreased PDCD4 protein expression is observed in $C M L$ patients. To evaluate PDCD4 protein expression levels in primary CML, PDCD4 protein expression in $50 \mathrm{CML}$ patients and 20 healthy controls was analyzed by western blot. The results demonstrated that all healthy controls exhibited high PDCD4 protein expression, however, PDCD4 protein expression was markedly decreased or absent in the $50 \mathrm{CML}$ samples, in accordance with that of PDCD4 mRNA (Fig. 2A). PDCD4 protein expression in CML patients was significantly decreased when compared with healthy controls $(\mathrm{P}<0.01$; Fig. 2B).

PDCD4 expression is significantly associated with BCR-ABL expression. BCR-ABL exhibits an important function in the development of CML, therefore the association between
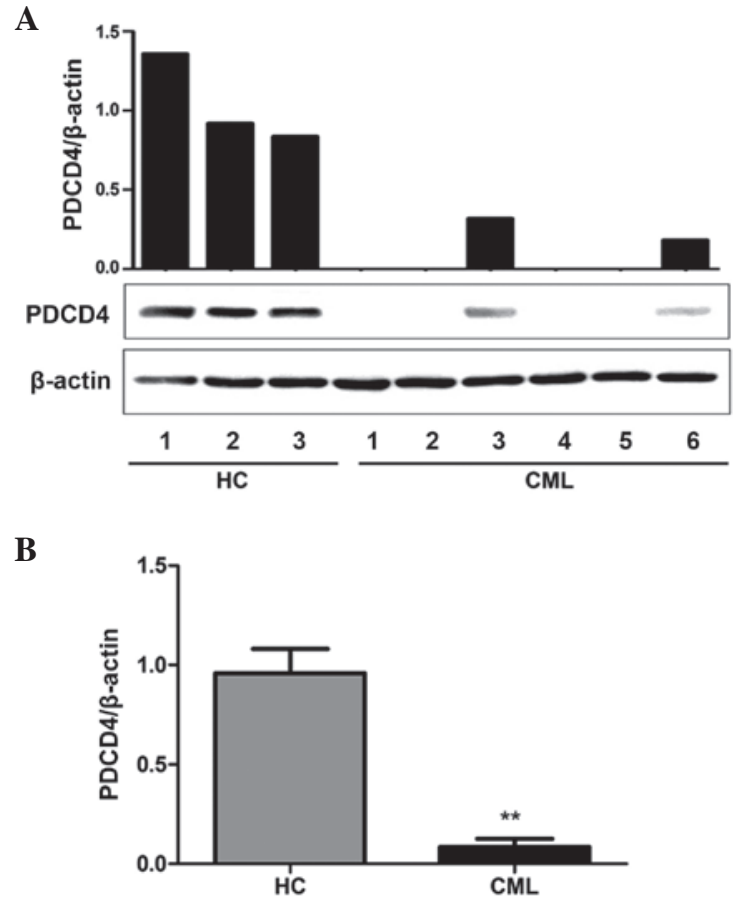

Figure 2. PDCD4 protein expression in 50 CML patients and $20 \mathrm{HCs}$ was evaluated by western blot analysis. (A) PDCD4 protein expression was higher in HCs compared with CML patients. Data were normalized to $\beta$-actin (CML lanes 1-3, CML-chronic phase; lanes 4 and 5, CML-accelerated phase; lane 6, CML-blast phase). (B) Normalized PDCD4 protein levels in 50 primary CML and $20 \mathrm{HCs}$ were analyzed. PDC4 expression in CML patients was significantly decreased compared with HCs. ${ }^{* *} \mathrm{P}<0.01$. PDCD4, programmed cell death 4; GAPDH, glyceraldehyde 3-phosphate dehydrogenase; CML, chronic myeloid leukemia; HC, healthy controls.

PDCD4 expression and BCR-ABL expression was analyzed by Pearson correlation analysis. As PDCD4 expression was markedly decreased or absent in CML patients, $20 \mathrm{CML}$ patients with relatively high levels of PDCD4 expression (PDCD4/GAPDH>0.1) were selected for the analysis. The results revealed that PDCD4 expression was negatively correlated with BCR-ABL expression $(n=20 ; r=-0.6716 ; \mathrm{P}<0.001$; Fig. 3), which indicates that PDCD4 expression is associated with the development and progression of CML. 


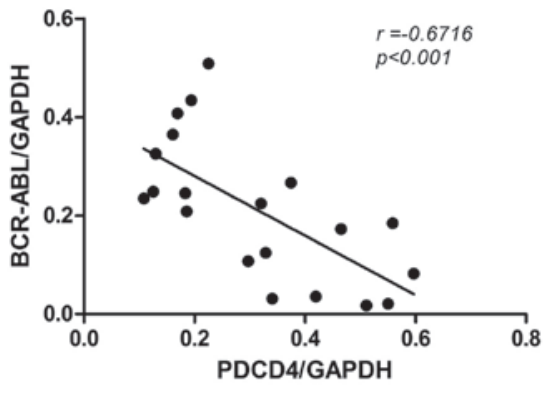

Figure 3. Association between PDCD4 and BCR-ABL expression. PDCD4 expression was negatively correlated with BCR-ABL expression ( $\mathrm{r}=-0.6716$; $\mathrm{P}<0.001$ ). PDCD4, programmed cell death 4; GAPDH, glyceraldehyde 3-phosphate dehydrogenase.

Imatinib increases PDCD4 levels in the K562 cell line. Since PDCD4 expression was negatively correlated with BCR-ABL expression, PDCD4 expression in K562 cells after 6, 24 and $48 \mathrm{~h}$ treatment with 0.5 and $1 \mu \mathrm{M}$ imatinib was analyzed by RT-qPCR and western blot analysis. The results revealed that the proliferation of K562 cells was significantly inhibited following $48 \mathrm{~h}$ of 0.5 and $1 \mu \mathrm{M}$ imatinib treatment $(\mathrm{P}<0.05$; Fig. 4A). Furthermore, PDCD4 mRNA expression was significantly increased $(\mathrm{P}<0.05$; Fig. 4B) and BCR-ABL expression $(\mathrm{P}<0.05$; Fig. $4 \mathrm{C})$ was significantly decreased after 24 and $48 \mathrm{~h}$ of treatment with 0.5 and $1 \mu \mathrm{M}$ imatinib. Western blot analysis of PDCD4 protein expression demonstrated the same results (Fig. 4D and E). These results indicated that decreased BCR-ABL expression may enhance PDCD4 expression and inhibit malignant proliferation of K562 cells.

\section{Discussion}

$\mathrm{CML}$ is characterized by the presence of the $\mathrm{Ph}$ chromosome, which results from a balanced reciprocal translocation between the ABL gene on chromosome 9 and the BCR gene on chromosome 22 (1). A number of tumor suppressor genes are inactivated or downregulated by BCR-ABL in $\mathrm{Ph}^{+}$leukemia, including protein phosphatase 2 (21), p53 (22) and phosphatase and tensin homolog (23). The results of the present study revealed that the tumor suppressor, PDCD4, is also downregulated in CML patients and is negatively correlated with BCR-ABL expression. In addition, inhibition of BCR-ABL expression by imatinib induced PDCD4 expression in the CML K562 cell line.

$P D C D 4$ was recently identified as a tumor suppressor gene and its expression is markedly decreased or absent in a number of solid tumors (9-13). However, the expression of PDCD4 in hematological neoplasms has rarely been reported. Recently, it was reported that miR-21 is frequently overexpressed in AML blasts, in association with a marked PDCD4 protein downmodulation in nucleophosmin-mutant acute myeloid leukemia (24). In the present study, PDCD4 mRNA and protein expression were significantly decreased in CML patients compared with healthy controls, which suggests that PDCD4 is involved in normal hematopoietic differentiation. PDCD4 is known to suppress protein translation by directly interacting with eIF4A to inhibit the formation of the translation-initiation complex (4) or by interfering with translation elongation via an RNA-binding domain (6), which indicates that PDCD4 inhibits cell proliferation

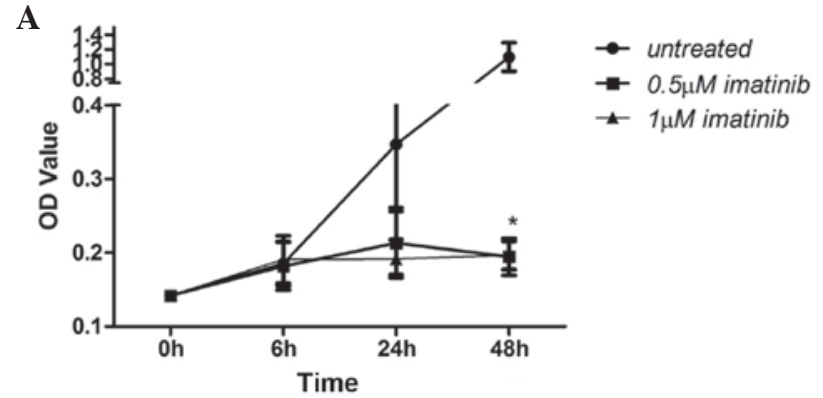

B

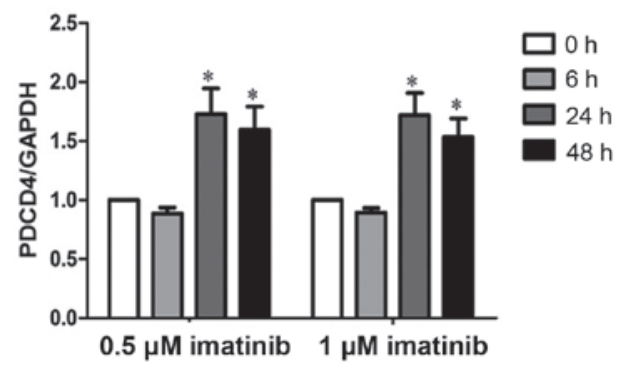

C

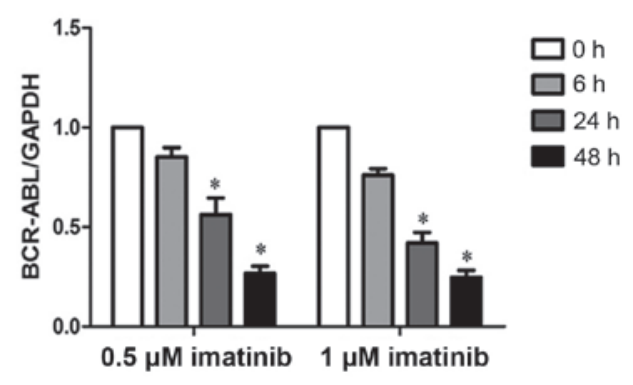

D

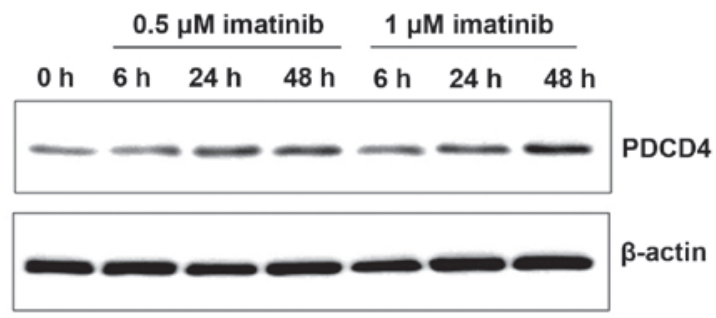

E

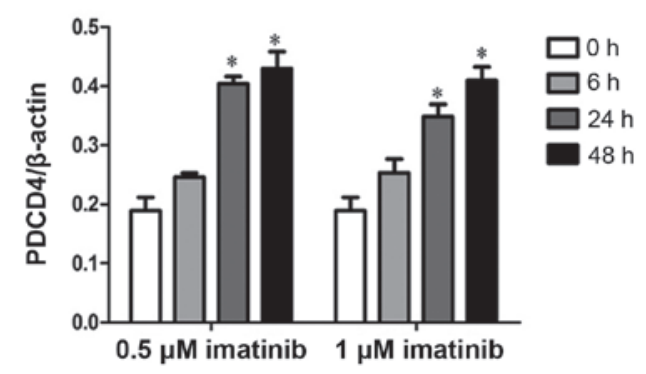

Figure 4. Imatinib increases PDCD4 expression in the K562 cell line. (A) MTT assay revealed that cell proliferation of K562 cells was decreased following treatment with 0.5 and $1 \mu \mathrm{M}$ imatinib for 6,24 and $48 \mathrm{~h}$. (B) RT-qPCR revealed (B) PDCD4 mRNA and expression was significantly increased and (C) BCR-ABL mRNA expression was significantly decreased in K562 cells following 24 and $48 \mathrm{~h}$ treatment with 0.5 and $1 \mu \mathrm{M}$ imatinib. (D) Western blot analysis revealed that PDCD4 protein expression was increased following treatment with 0.5 and $1 \mu \mathrm{M}$ imatinib in $\mathrm{K} 562$ cells. (E) PDCD4 protein levels were normalized to $\beta$-actin, which revealed that PDCD4 protein levels were significantly increased following treatment with 0.5 and $1 \mu \mathrm{M}$ for 24 and $48 \mathrm{~h}$ in K562 cells. All data are expressed as the mean of triplicate experiments \pm standard error of the mean. ${ }^{*} \mathrm{P}<0.05$ vs. untreated cells. PDCD4, programmed cell death 4; GAPDH, glyceraldehyde 3-phosphate dehydrogenase. 
and is involved in tumor development and progression. Furthermore, PDCD4 also suppresses autophagy and promotes cell differentiation (25). In addition, it has been reported that PDCD4 expression contributes to all-trans retinoic acid (ATRA)-induced granulocytic but not monocytic/macrophagic differentiation in acute myeloid leukemia, and ATRA induces PDCD4 expression via the inhibition of phosphoinositide 3-kinase (PI3K)/ protein kinase $\mathrm{B}(\mathrm{Akt}) /$ mammalian target of rapamycin (mTOR) pathway (26). PDCD4 is also involved in the germ line stem cell differentiation pathway: It is hypothesized to relieve the inhibition of Bam by eIF4A (27). These findings suggest that PDCD4 may contribute to cell differentiation of CML. However, in the present study, no significant difference in PDCD4 expression was observed between CML-CP, CML-AP and CML-BP patients, which indicates that PDCD4 does not promote the transition of CML from CP to BP. However, a recent study demonstrated that in the blast crisis of CML, RBP2 expression was decreased; moreover, RBP2 could induce cell differentiation and inhibit cell proliferation, which depend on inhibiting miR-21 and increasing PDCD4 expression (28). All these findings suggest that PDCD4 may play a role in the blast crisis of CML.

The BCR-ABL oncoprotein constitutively activates several downstream pathways (29). One important target is the $\mathrm{PI} 3 \mathrm{~K} / \mathrm{AKT} / \mathrm{mTor}$ pathway, which is constitutively activated in BCR-ABL-transformed cells and is inhibited by imatinib mesylate (30). In this study, the expression of PDCD4 and BCR-ABL in primary CML patients was investigated, which revealed that PDCD4 expression was negatively correlated with BCR-ABL expression. In addition, following 24 and $48 \mathrm{~h}$ of imatinib treatment, PDCD4 expression was significantly upregulated in K562 cells. These results suggest that BCR-ABL negatively regulates PDCD4 expression in CML patients, which is consistent with the results of previous studies, which reported that inhibition of the BCR-ABL/mTOR/p70 S6K pathway results in enhanced protein expression of PDCD4 in K562 cells (31) and inhibition of p70 S6 kinase activity by fluvastatin results in the upregulation of expression of PDCD4 in renal cell carcinoma (32).

In conclusion, the results of the present study indicate that the downregulation of PDCD4 expression is associated with the development of human CML. Therefore, increased understanding with regard to the involvement of PDCD4 in CML progression may provide novel molecular targets for diagnosis and therapy of human CML.

\section{Acknowledgements}

This study was supported by The National Natural Science Foundation of China (grant nos. 81202069 and 81470403) and The Yantai Science and Technology Development Plan (grant no. 2012071).

\section{References}

1. Quintás-Cardama A and Cortes JE: Chronic myeloid leukemia: Diagnosis and treatment. Mayo Clin Proc 81: 973-988, 2006.

2. Quintás-Cardama A and Cortes JE: Molecular biology of bcr-abl1-positive chronic myeloid leukemia. Blood 113: 1619-1630, 2009.

3. Perrotti D, Jamieson C, Goldman J and Skorski T: Chronic myeloid leukemia: Mechanisms of blastic transformation. J Clin Invest 120: 2254-2264, 2010.
4. Yang HS, Cho MH, Zakowicz H, Hegamyer G, Sonenberg N and Colburn NH: A novel function of the MA-3 domains in transformation and translation suppressor Pdcd4 is essential for its binding to eukaryotic translation initiation factor 4A. Mol Cell Biol 24: 3894-3906, 2004

5. Singh P, Wedeken L, Waters LC, Carr MD and Klempnauer KH: Pdcd4 directly binds the coding region of c-myb mRNA and suppresses its translation. Oncogene 30: 4864-4873, 2011.

6. Biyanee A, Ohnheiser J, Singh P and Klempnauer KH: A novel mechanism for the control of translation of specific mRNAs by tumor suppressor protein Pdcd4: Inhibition of translation elongation. Oncogene 34: 1384-1392, 2015.

7. Hilliard A, Hilliard B, Zheng SJ, Sun H, Miwa T, Song W, Göke R and Chen YH: Translational regulation of autoimmune inflammation and lymphoma genesis by programmed cell death 4. J Immunol 177: 8095-8102, 2006.

8. Jansen AP, Camalier CE and Colburn NH: Epidermal expression of the translation inhibitor programmed cell death 4 suppresses tumorigenesis. Cancer Res 65: 6034-6041, 2005.

9. Chen Y, Knösel T, Kristiansen G, Pietas A, Garber ME, Matsuhashi S, Ozaki I and Petersen I: Loss of PDCD4 expression in human lung cancer correlates with tumour progression and prognosis. J Pathol 200: 640-646, 2003.

10. Zhang $\mathrm{H}$, Ozaki I, Mizuta T, Hamajima H, Yasutake T, Eguchi Y, Ideguchi H, Yamamoto K and Matsuhashi S: Involvement of programmed cell death 4 in transforming growth factor-betal-induced apoptosis in human hepatocellular carcinoma. Oncogene 25: 6101-6112, 2006.

11. Mudduluru G, Medved F, Grobholz R, Jost C, Gruber A, Leupold JH, Post S, Jansen A, Colburn NH and Allgayer H: Loss of programmed cell death 4 expression marks adenoma-carcinoma transition, correlates inversely with phosphorylated protein kinase $\mathrm{B}$, and is an independent prognostic factor in resected colorectal cancer. Cancer 110: 1697-1707, 2007.

12. Wei ZT, Zhang X, Wang XY, Gao F, Zhou CJ, Zhu FL, Wang Q, Gao Q, Ma CH, Sun WS, et al: PDCD4 inhibits the malignant phenotype of ovarian cancer cells. Cancer Sci 100: 1408-1413, 2009.

13. Gao F, Zhang P, Zhou C, Li J, Wang Q, Zhu F, Ma C, Sun W and Zhang L: Frequent loss of PDCD4 expression in human glioma: Possible role in the tumorigenesis of glioma. Oncol Rep 17: 123-128, 2007.

14. Yang HS, Knies JL, Stark C and Colburn NH: Pdcd4 suppresses tumor phenotype in JB6 cells by inhibiting AP-1 transactivation. Oncogene 22: 3712-3720, 2003.

15. Yang HS, Matthews CP, Clair T, Wang Q, Baker AR, Li CC, Tan TH and Colburn NH: Tumorigenesis suppressor Pdcd4 down-regulates mitogen-activated protein kinase kinase kinase kinase 1 expression to suppress colon carcinoma cell invasion. Mol Cell Biol 26: 1297-1306, 2006.

16. Leupold JH, Yang HS, Colburn NH, Asangani I, Post S and Allgayer H: Tumor suppressor Pdcd4 inhibits invasion/intravasation and regulates urokinase receptor (u-PAR) gene expression via Sp-transcription factors. Oncogene 26: 4550-4562, 2007.

17. Wang Q, Sun Z and Yang HS: Downregulation of tumor suppressor Pdcd 4 promotes invasion and activates both beta-catenin/Tcf and AP-1-dependent transcription in colon carcinoma cells. Oncogene 27: 1527-1535, 2008.

18. Wang Q, Sun ZX, Allgayer H and Yang HS: Downregulation of E-cadherin is an essential event in activating beta-catenin/Tcf-dependent transcription and expression of its target genes in Pdcd4 knockdown cells. Oncogene 29: 128-138, 2010.

19. Vardiman JW, Harris NL and Brunning RD: The World Health Organization (WHO) classification of the myeloid neoplasms. Blood 100: 2292-2302, 2002.

20. Livak KJ and Schmittgen TD: Analysis of relative gene expression data using real-time quantitative PCR and the 2(-Delta Delta C(T)) Method. Methods 25: 402-408, 2001

21. Neviani P, Santhanam R, Trotta R, Notari M, Blaser BW, Liu S, Mao H, Chang JS, Galietta A, Uttam A, et al: The tumor suppressor PP2A is functionally inactivated in blast crisis CML through the inhibitory activity of the BCR/ABL-regulated SET protein. Cancer Cell 8: 355-368, 2005.

22. Beck Z, Kiss A, Tóth FD, Szabó J, Bácsi A, Balogh E, Borbély A, Telek B, Kovács E, Oláh E and Rak K: Alterations of P53 and RB genes and the evolution of the accelerated phase of chronic myeloid leukemia. Leuk Lymphoma 38: 587-597, 2000 . 
23. Peng C, Chen Y, Yang Z, Zhang H, Osterby L, Rosmarin AG and Li S: PTEN is a tumor suppressor in CML stem cells and BCR-ABL-induced leukemias in mice. Blood 115: 626-635, 2010.

24. Riccioni R, Lulli V, Castelli G, Biffoni M, Tiberio R, Pelosi E, Lo-Coco F and Testa U: miR-21 is overexpressed in NPM1-mutant acute myeloid leukemias. Leuk Res 39: 221-228, 2015.

25. Song X, Zhang X, Wang X, Zhu F, Guo C, Wang Q, Shi Y, Wang J, Chen Y and Zhang L: Tumor suppressor gene PDCD4 negatively regulates autophagy by inhibiting the expression of autophagy-related gene ATG5. Autophagy 9: 743-755, 2013.

26. Ozpolat B, Akar U, Steiner M, Zorrilla-Calancha I, Tirado-Gomez M, Colburn N, Danilenko M, Kornblau S and Berestein GL: Programmed cell death-4 tumor suppressor protein contributes to retinoic acid-induced terminal granulocytic differentiation of human myeloid leukemia cells. Mol Cancer Res 5: 95-108, 2007.

27. Cash AC and Andrews J: Fine scale analysis of gene expression in Drosophila melanogaster gonads reveals Programmed cell death 4 promotes the differentiation of female germline stem cells. BMC Dev Biol 12: 4, 2012.
28. Zhou M, Zeng J, Wang X, Wang X, Huang T, Fu Y, Sun T, Jia J and Chen C: Histone demethylase RBP2 decreases miR-21 in blast crisis of chronic myeloid leukemia. Oncotarget 6: 1249-1261, 2015.

29. Cilloni D and Saglio G: Molecular pathways: BCR-ABL. Clin Cancer Res 18: 930-937, 2012.

30. Parmar S, Smith J, Sassano A, Uddin S, Katsoulidis E, Majchrzak B, Kambhampati S, Eklund EA, Tallman MS, Fish EN and Platanias LC: Differential regulation of the p70 S6 kinase pathway by interferon alpha (IFNalpha) and imatinib mesylate (STI571) in chronic myelogenous leukemia cells. Blood 106: 2436-2443, 2005.

31. Carayol N, Katsoulidis E, Sassano A, Altman JK, Druker BJ and Platanias LC: Suppression of programmed cell death 4 (PDCD4) protein expression by BCR-ABL-regulated engagement of themTOR/p70 S6 kinase pathway. J Biol Chem 283: 8601-8610, 2008.

32. Woodard J, Sassano A, Hay N and Platanias LC: Statin-dependent suppression of the $\mathrm{Akt} / \mathrm{mammalian}$ target of rapamycin signaling cascade and programmed cell death 4 up-regulation in renal cell carcinoma. Clin Cancer Res 14: 4640-4649, 2008. 